\title{
Gigantismo e acromegalia causati da microduplicazioni del locus Xp26 e da mutazione del gene GPR101
}

\author{
Salvatore Cannavò
}

Pubblicato online: 17 gennaio 2015

(C) Springer International Publishing AG 2015

Commento a:

Gigantism and acromegaly due to Xp26 microduplications and GPR101 mutation.

G. Trivellin, A.F. Daly, F.R. Faucz, B. Yuan,

L. Rostomyan, D.O. Larco, M.H. Schernthaner-Reiter,

E. Szarek, L.F. Leal, J.-H. Caberg, E. Castermans,

C. Villa, A. Dimopoulos, P. Chittiboina, P. Xekouki,

N. Shah, D. Metzger, P.A. Lysy, E. Ferrante,

N. Strebkova, N. Mazerkina, M.C. Zatelli, M. Lodish,

A. Horvath, R. Bertollo de Alexandre, A.D. Manning,

I. Levy, M.F. Keil, M. de la Luz Sierra, L. Palmeira,

W. Coppieters, M. Georges, L.A. Naves, M. Jamar,

V. Bours, T.J. Wu, C.S. Choong, J. Bertherat,

P. Chanson, P. Kamenický, W.E. Farrell, A. Barlier,

M. Quezado, I. Bjelobaba, S.S. Stojilkovic, J. Wess,

S. Costanzi, P. Liu, J.R. Lupski, A. Beckers,

C.A. Stratakis.

New Engl J Med (2014) 371(25):2363-2374

In un recentissimo studio multicentrico pubblicato sul $\mathrm{New}$ England Journal of Medicine, Trivellin et al. hanno dimostrato la presenza di microduplicazioni del locus Xq26.3 in $11(32 \%)$ su 43 pazienti con acrogigantismo non sindromico insorto in età pediatrica, ma negativi per mutazioni del gene AIP, proponendo per tale condizione la definizione di $X$-linked acrogigantism (X-LAG). In 10 di essi era evidente un adenoma ipofisario, mentre in un caso l'esame istologico è risultato conclusivo per iperplasia ipofisaria. I pazienti $\mathrm{X}$-LAG si caratterizzerebbero per una più precoce insorgenza di malattia (0,5-2 anni), e quindi anche per una maggiore

S. Cannavò ( $\varangle)$

Dipartimento di Medicina Clinica e Sperimentale, Università di

Messina, Messina, Italia

e-mail: cannavos@unime.it precocità della diagnosi, più elevati livelli sierici di IGF-1 e maggiore prevalenza di iperprolattinemia rispetto agli altri casi di acrogigantismo insorto in età pediatrica ma senza microduplicazioni di Xq26. Il 71\% dei pazienti X-LAG erano di sesso femminile, contro il $24 \%$ nel restante gruppo. Inoltre, solo il $36 \%$ dei pazienti X-LAG, ma nessuno degli altri, presentava familiarità per adenomi ipofisari isolati (FIPA).

Nella regione duplicata del locus Xq26.3 risiedono 4 geni di cui uno, GPR101, codifica per un recettore orfano accoppiato a una proteina-G stimolatoria. Tale gene si candida come responsabile della malattia, in virtù della capacità di indurre la stimolazione dell'adenil-ciclasi con potenziali effetti patogenetici simili a quelli indotti dalle mutazioni di GNAS. In effetti, il gene GPR101 è risultato estremamente attivato negli adenomi ipofisari dei pazienti X-LAG. Inoltre, l'analisi immunoistochimica ha dimostrato l'iperespressione della proteina codificata da tale gene nel tumore ipofisario di alcuni pazienti.

Le microduplicazioni del locus Xq26 non sono state identificate in nessuno dei 243 pazienti adulti con acromegalia sporadica esaminati nello stesso studio. Tuttavia, una mutazione del gene GPR101 che causa una sostituzione c.924G $\rightarrow$ C (p.E308D) è stata identificata in 11 di essi $(4,4 \%)$. In 3 casi tale mutazione era germinale, mentre negli altri casi era solo somatica. Nessuna mutazione del gene GPR101 è stata comunque individuata in 13 famiglie FIPA, così come in 7600 controlli.

In conclusione, Trivellin et al. hanno dimostrato che le microduplicazioni del locus Xq26 causano l'insorgenza precocissima di acrogigantismo, verosimilmente inducendo l'iperespressione del gene GPR101. Inoltre, una mutazione attivante il gene GPR101 è stata individuata nel 4,4\% dei pazienti adulti con acromegalia sporadica. 